\begin{tabular}{|c|l|}
\hline Title & Transcription factor 8 activates R-Ras to regulate angiogenesis \\
\hline Author(s) & Inuzuka, Takayuki; Tsuda, Masumi; Kawaguchi, Hideaki; Ohba, Y usuke \\
\hline Citation & $\begin{array}{l}\text { Biochemical and Biophysical Research Communications, 379(2), 510-513 } \\
\text { https://doi.org/10.1016/.bbrc.2008.12.101 }\end{array}$ \\
\hline Issue Date & 2009-02-06 \\
\hline Doc URL & http://hdl.handle.net/2115/54566 \\
\hline Type & article (author version) \\
\hline File Information & Biochem Biophys Res Commun_379(2)_510-513.pdf \\
\hline
\end{tabular}

Instructions for use 


\title{
Transcription factor 8 activates R-Ras to regulate angiogenesis
}

\author{
Takayuki Inuzuka, Masumi Tsuda, Hideaki Kawaguchi, and Yusuke Ohba* \\ Laboratory of Pathophysiology and Signal Transduction, \\ Hokkaido University Graduate School of Medicine, \\ N15W7, Kita-ku, Sapporo 060-8638, Japan
}

Correspondence author. Fax: +81-11-706-7877.

E-mail address: yohba@med.hokudai.ac.jp (Y. Ohba). 


\begin{abstract}
We have recently reported that transcription factor 8 (TCF8) negatively regulates pathological angiogenesis by regulating endothelial invasiveness by acting as a transcriptional attenuator of matrix metalloproteinase 1. TCF8 also modulates cell-matrix and cell-cell adhesion; however molecular mechanism of this TCF8 function remains obscure. Here, we provide evidence that TCF8 activates R-Ras, another class of angiogenic regulator, to suppress angiogenesis by a mechanism other than a transcriptional attenuator. Tube formation by human umbilical vein endothelial cells (HUVECs) facilitated by TCF8 suppression was significantly inhibited by the expression of constitutive active mutant of RRas. When we examined the mRNA expression levels of R-Ras regulators, no significant changes were observed to explain the R-Ras activation by TCF8. Interestingly, we found that TCF8 bound to CalDAG-GEFIII, an R-Ras activator, in the cytosol, indicating that TCF8 emanates signaling for R-Ras activation from cytosol to regulate angiogenesis negatively.
\end{abstract}

Keywords: TCF8; R-Ras; CalDAG-GEF; angiogenesis 


\section{Introduction}

The two-handed zinc finger (ZF) homeodomain transcription factor TCF8, alternatively designated as $\delta \mathrm{EF} 1, \mathrm{ZEB}-1$, Nil2, AREB6, and Zfhxp1, has been implicated in a variety of biological phenomena, including cancer invasion, cellular senescence, viral infection, bone metabolism, T-cell and neuronal development, as well as pathological responses of blood vessels [1-6]. Most of these functions are ascribed to the transcriptional attenuation via the two ZFdomain clusters of TCF8; however, knowledge about the other function than transcriptional regulation is currently limited.

R-Ras, a member of Ras family of GTP-binding proteins, is originally isolated by lowstringency hybridization with a v-H-ras probe [7]. Apart from the similarity to the functions of classical Ras, such as the activation of the extracellular signal-regulated kinase/mitogenactivated protein kinase cascade[8], R-Ras seems to have its unique functions: stimulating cell adhesion by integrin [9], inhibiting apoptosis [10], and regulation of exocytosis [11]. However, so far, no specific effector molecules for R-Ras have been reported to explain its different (distinct) functions. Recently, R-Ras is implicated in pathological angiogenesis [12, 13], although mechanism of the specific participation of this molecule is yet to be determined.

Recently, we have reported that TCF8, of which expression is induced in endothelial cells during angiogenesis, negatively regulates tumor angiogenesis via transcriptional attenuation of matrix metalloprotease (MMP)-1, and via the augmentation of cell-matrix and cell-cell adhesion. However, molecular mechanism by which TCF8 regulates cell adhesiveness remains obscure. Here, we show that TCF8 activates R-Ras and provide evidence that the activation is due to the direct binding of TCF8 to CalDAG-GEFIII, an activator of R-Ras, but not transcriptional regulation of the R-Ras regulators. Our finding may explain how the TCF8-R-Ras axis specifically plays a role in pathological angiogenesis. 


\section{Materials and Methods}

Reagents and antibodies. Antibodies against TCF8 (ZEB1), HA, R-Ras, Flag were purchased from Santa Cruz Biotechnology (Santa Cruz, CA), Roche (Indianapolis, IN), Cell Signaling Technology (Beverly, MA), Stratagene (La Jolla, CA) respectively. The rabbit antiserum raised against CalDAG-GEFIII was kindly provided by Dr. M. Matsuda (Kyoto Univ., Kyoto, Japan).

Expression plasmids. Human TCF8 cDNA was obtained by RT-PCR of total RNA from HUVECs and was cloned into pCR-BluntII-TOPO (Invitrogen, Carlsbad, CA). To generate Flagor HA-tagged TCF8 expression vectors, the cDNA was subcloned into the XhoI and NotI sites of the pCXN2-Flag or pCXN2-3xHA vectors [14]. A series of expression vectors for TCF8 deletion mutants were constructed as above after PCR amplification with specific primers. All constructs were confirmed by sequencing analysis. pCXN2-Flag-R-Ras WT and pCXN2-Flag-CalDAGGEFI, II, and III were described previously [15]. The expression vectors for TCF8-CFP and YFP-CalDAGIII were generated as described above using pCXN2-Flag-CFPC and pCXN2YFPN. The self-inactivating (SIN) lentiviral vector pCS-CA-MCS, the packaging vector pCAGHIVgp, and the VSV-G- and REV-expressing construct pCMV-VSV-G-RSV-REV were obtained from Dr. H. Miyoshi (RIKEN, Tsukuba, Japan). After elimination of the NotI site within the irrelevant backbone, cDNA of Flag-R-RasV38 was subcloned into the EcoRI/NotI site of pCS-CA-MCS.

Cell culture and transfection. Human umbilical vein endothelial cells (HUVECs), which had been passaged 4 to 10 time, were maintained in complete endothelial basal medium (EBM2), and transfected with siRNA targeting the human TCF8 mRNA [si TCF8, [16]] using HiPerFect Transfection Reagent (QIAGEN, Valencia, CA) according to the manufacturer's instructions. AllStars Negative Control siRNA (si Control, QIAGEN) was used as a control. The cells were analyzed after 48 or $72 \mathrm{~h}$. HeLa and 293T cells were cultured in DMEM (Sigma, St. Louis, MO) supplemented with 10\% FCS. Expression plasmids were introduced with FuGENE 
HD (Roche) according to the manufacturer's protocol. Lentivirus-mediated gene transfer was performed as described previously [17].

Immunoblotting and immunoprecipitation. Cells were lysed in RIPA buffer [50 mM Tris-HCl, pH 7.4, $150 \mathrm{mM} \mathrm{NaCl,} 1 \mathrm{mM}$ EDTA, 1\% NP-40, 0.1\% SDS, 0.5\% sodium deoxycholate, $1 \mathrm{mM} \mathrm{Na}_{3} \mathrm{VO}_{4}$, and complete EDTA-free protease inhibitor (Roche)] for $20 \mathrm{~min}$ on ice and clarified by centrifugation. The supernatants were subjected to SDS-PAGE, and separated proteins were blotted on polyvinylidene difluoride membranes (Bio-Rad, Hercules, CA). Signals were developed by ECL Western Blotting Detection Reagent (GE Healthcare, Little Chalfont, UK) and detected using an LAS-1000UVmini image analyzer (FUJIFILM, Tokyo, Japan).

HUVECs were lysed in lysis buffer [10 mM Tris-HCl, pH 7.4, $150 \mathrm{mM} \mathrm{NaCl,} 5 \mathrm{mM}$ EDTA, $0.5 \%$ NP-40, $10 \%$ glycerol, $1 \mathrm{mM} \mathrm{Na}_{3} \mathrm{VO}_{4}$, and complete EDTA-free protease inhibitor (Roche)], precipitated with anti-CalDAGIII antisera and protein A-Sepharose. Proteins bound to beads were separated by SDS-PAGE, and detected by immunoblotting.

Pull-down assay for R-Ras. Detection of GTP-bound R-Ras was performed essentially as described previously [18]. Briefly, cells were lysed in lysis buffer (50 mM Tris-HCl, $\mathrm{pH} 7.4,150$ $\mathrm{mM} \mathrm{NaCl}, 5 \mathrm{mM} \mathrm{MgCl} 2,1 \% \mathrm{NP}-40,0.5 \%$ sodium deoxycholate, $0.1 \% \mathrm{SDS}$, and $1 \mathrm{mM}$ $\mathrm{Na}_{3} \mathrm{VO}_{4}$ ), clarified by centrifugation, and incubated with GST-RalGDS-RBD for $30 \mathrm{~min}$. The resulting complexes of GTP-bound R-Ras and GST-RalGDS-RBD were precipitated with glutathione-Sepharose beads, washed twice with lysis buffer, and eluted. GTP-bound R-Ras was detected by immunoblotting.

$R N A$ isolation and RT-PCR. RNA isolation and quantitative RT-PCR were performed as described previously $[6,19]$. The primers used for PCR amplification are listed in Supplementary Table. Data were normalized by the expression level of GAPDH in each sample. 
Fluorescence microscopy. Immunofluorescence microscopy and intermolecular FRET analysis were performed as described [20,21].

Tube formation assay. Tube formation by HUVECs on Matrigel (BD-Discovery Labware, Bedford, MA) was performed as described previously [22, 23] with some modifications. Each well of a 48-well plate was coated with $125 \mu \mathrm{lof} 10 \mathrm{mg} / \mathrm{ml}$ Matrigel, and was filled with $2 \times 10^{4}$ cells in $500 \mu$ of complete EGM-2. At the indicated times, ten images of the formed tubes were obtained for every well and the number of tubes was quantified.

Statistical analyses. All data represent means and SD of experiments performed in triplicate, and were subjected to one-way analysis of variance, followed by the comparison by Student's $t$-tests. $P$ values obtained from the tests are described in the figure legends. 


\section{Results and Discussion}

We first tested whether TCF8 is involved in R-Ras signaling. R-Ras activity in the quiescent human umbilical endothelial cells (HUVECs) is lower than the detectable level and the effect of TCF8 knockdown on R-Ras activation could not be observed (data not shown); however, cell adhesion-induced R-Ras activation was substantially inhibited in TCF8-silenced HUVECs (Fig. 1A). Conversely, overexpression of TCF8 dramatically activated R-Ras in 293T cells (Fig. 1B). Next, the functional importance of R-Ras as the effector of TCF8 was examined by utilizing lentivirus-mediated gene transfer [17], which yields sufficient protein expression with approximately 70\% transfection efficiency in HUVECs (Fig. 1C). Expression of R-Ras V38, the constitutive active mutant of R-Ras, attenuated the enhanced tube formation obtained with TCF8-suppressed HUVECs (Fig. 1D and E). These data thereby indicate that TCF8 mediates RRas signaling in the negative regulation of angiogenesis.

Next, to explore the detailed mechanism through which TCF8 regulates R-Ras, we generated deletion mutants of TCF8 (Fig. 2A), and found that TCF8-HDCZF and TCF8-CZF, but not TCF8-NZF or TCF8-HD, activated R-Ras, suggesting that a cluster of C-terminal ZF domains is essential for R-Ras activation (Fig. 2B). Since the C-terminal ZF domains are required for the canonical function of TCF8 to bind to the E2-box sequence CACCTG on target genes and to regulate their transcription [24], we examined the effect of TCF8 on mRNA levels of R-Ras regulators, including guanine nucleotide exchange factors (GEFs) and GTPaseactivating proteins (GAPs). RT-PCR revealed no significant differences in the expression of these proteins, which would reflect R-Ras activation by TCF8 (Fig. 2C). Essentially similar results were obtained when we used 293T cells (Fig. 2D). This is an unexpected result because most previous works have shown the significance of transcriptional attenuation of target genes in the explanation of the phenotypes obtained. Although, of course, TCF8 can be involved in the transcriptional regulation of other class of regulators than GEFs and GAPs, it is possible that TCF8 activates R-Ras by different mechanism from transcriptional attenuator. 
During the course of experiments to examine the effect of TCF8 on the mRNA expression of GEFs and GAPs in Fig. 2, we noticed that, of the calcium- and diacylglycerol (DAG)-responding GEFs, CalDAG-GEFIII was preferentially expressed in HUVECs, as was previously reported [25]. Therefore, we hypothesized that TCF8 mediates CalDAG-GEFIII activation by the direct interaction. For this purpose, we utilized antiserum specific for CalDAGGEFIII (Fig. 3A) to determine whether TCF8 bound to CalDAG-GEFIII. Coimmunoprecipitation analysis revealed an interaction between endogenous TCF8 and CalDAGGEFIII in HUVECs (Fig. 3B). In addition, co-expression of TCF8 and CalDAGIII achieved an additive effect on R-Ras activation (Fig. 3C). Localization studies of TCF8 and its truncated mutants revealed that R-Ras-activating proteins, TCF8-FL, TCF8-HDCZF, and TCF8-CZF, localized to both the nucleus and the cytoplasm, whereas the proteins lacking the ability to activate R-Ras, TCF8-NZF and TCF8-HD, were restrictively localized in the nucleus and at the plasma membrane, respectively (Fig. 3D). Furthermore, the interaction of TCF8 and CalDAGIII was observed in the cytoplasm by fluorescence resonance energy transfer (FRET) (Fig. 3E). Although the exact mechanism of how TCF8 activates CalDAGIII remains obscure, we provide evidence for a novel function for TCF8 in the cytoplasm, beyond transcriptional regulation.

In the present study, we show that TCF8 activates R-Ras though the distinct function than transcription attenuator. The pull-down analysis using the truncated mutants revealed that the Cterminal ZF domains of TCF8 are required for R-Ras activation (Fig. 2B). Furthermore, the localization studies revealed that the proteins activating R-Ras resided in, and are bound to CalDAGIII in the cytosol (Fig. 3D and E), suggesting a novel cytoplasmic function for TCF8. Interestingly, it was previously reported that mice lacking the 728-1124 aa that include the Cterminal ZF domains of TCF8 can survive, unlike mice deficient in full length TCF8 [4]. With respect to the importance of these C-terminal ZF domains in the activation of R-Ras, it is noteworthy that R-Ras KO mice are healthy under normal conditions [12], suggesting that the molecular mechanism involved in developmental stages are quite distinct from those needed for 
pathological angiogenesis, which apparently requires the TCF8 C-terminal ZF domains and downstream R-Ras activation $[6,12]$.

The communication between endothelial cells and ECMs is crucial for angiogenesis [22, 26], which are attenuated in TCF8-repressed HUVECs [6]. The depletion of TCF8 significantly hampers cell adhesion-induced activation of R-Ras (Fig. 1A). Therefore, R-Ras can participate in the TCF8 mediated signaling pathway to cell-matrix adhesion, and subsequent angiogenesis (Fig. $1 \mathrm{D}$ and $\mathrm{E})$. The presented data here may provide novel acceptable regulatory mechanism to explain R-Ras specific function. Furthermore, our findings on the restricted expression pattern of TCF8 within tumor vessels [6] may therefore account for the specific implication of R-Ras in the negative regulation of pathological angiogenesis [12]. 


\section{Acknowledgements}

We thank M. Matsuda for plasmids and antisera against CalDAG-GEFIII, H. Miyoshi for lentivirus vectors, K. Hida for providing cells, N. Toyoda for technical assistance, S. Darmanin for critical reading of the manuscript, and members of our laboratory for helpful discussions. This work is supported in part by Grants-in-Aid for Scientific Research from the Ministry of Education, Culture, Sports, Science and Technology, Japan, and Japan Society for the Promotion of Science (JSPS), and by grants from the Uehara Memorial Foundation and Mochida Memorial Foundation for Medical and Pharmaceutical Research. T.I. is a JSPS fellow. 


\section{REFERENCES}

[1] H. Peinado, D. Olmeda, A. Cano, Snail, Zeb and bHLH factors in tumour progression: an alliance against the epithelial phenotype? Nat. Rev. Cancer 7 (2007) 415-428.

[2] A. Zwijsen, L. A. van Grunsven, E. A. Bosman, C. Collart, L. Nelles, L. Umans, T. Van de Putte, G. Wuytens, D. Huylebroeck, K. Verschueren, Transforming growth factor beta signalling in vitro and in vivo: activin ligand-receptor interaction, Smad5 in vasculogenesis, and repression of target genes by the deltaEF1/ZEB-related SIP1 in the vertebrate embryo, Mol. Cell. Endocrinol. 180 (2001) 13-24.

[3] T. Takagi, H. Moribe, H. Kondoh, Y. Higashi, DeltaEF1, a zinc finger and homeodomain transcription factor, is required for skeleton patterning in multiple lineages, Development 125 (1998) 21-31.

[4] Y. Higashi, H. Moribe, T. Takagi, R. Sekido, K. Kawakami, H. Kikutani, H. Kondoh, Impairment of T cell development in deltaEF1 mutant mice, J. Exp. Med. 185 (1997) 14671479.

[5] G. Nishimura, I. Manabe, K. Tsushima, K. Fujiu, Y. Oishi, Y. Imai, K. Maemura, M. Miyagishi, Y. Higashi, H. Kondoh, R. Nagai, DeltaEF1 mediates TGF-beta signaling in vascular smooth muscle cell differentiation, Dev. Cell 11 (2006) 93-104.

[6] T. Inuzuka, M. Tsuda, S. Tanaka, H. Kawaguchi, Y. Higashi, Y. Ohba, Integral role of TCF8 in the negative regulation of tumor angiogenesis, Cancer Res. (in press).

[7] D. G. Lowe, D. J. Capon, E. Delwart, A. Y. Sakaguchi, S. L. Naylor, D. V. Goeddel, Structure of the human and murine R-ras genes, novel genes closely related to ras protooncogenes, Cell 48 (1987) 137-146.

[8] A. D. Cox, T. R. Brtva, D. G. Lowe, C. J. Der, R-Ras induces malignant, but not morphologic, transformation of NIH3T3 cells, Oncogene 9 (1994) 3281-3288.

[9] Z. Zhang, K. Vuori, H. Wang, J. C. Reed, E. Ruoslahti, Integrin activation by R-ras, Cell 85 (1996) 61-69. 
[10] J. Suzuki, Y. Kaziro, H. Koide, An activated mutant of R-Ras inhibits cell death caused by cytokine deprivation in BaF3 cells in the presence of IGF-I, Oncogene 15 (1997) 16891697.

[11] A. Takaya, T. Kamio, M. Masuda, N. Mochizuki, H. Sawa, M. Sato, K. Nagashima, A. Mizutani, A. Matsuno, E. Kiyokawa, M. Matsuda, R-Ras regulates exocytosis by Rgl2/Rlfmediated activation of RalA on endosomes, Mol. Biol. Cell 18 (2007) 1850-1860.

[12] M. Komatsu, E. Ruoslahti, R-Ras is a global regulator of vascular regeneration that suppresses intimal hyperplasia and tumor angiogenesis, Nat. Med .11 (2005) 1346-1350.

[13] S. Iwashita, M. Kobayashi, Y. Kubo, Y. Hinohara, M. Sezaki, K. Nakamura, R. SuzukiMigishima, M. Yokoyama, S. Sato, M. Fukuda, M. Ohba, C. Kato, E. Adachi, S. Y. Song, Versatile roles of R-Ras GAP in neurite formation of PC12 cells and embryonic vascular development, J. Biol. Chem. 282 (2007) 3413-3417.

[14] K. Terai, M. Matsuda, Ras binding opens c-Raf to expose the docking site for mitogenactivated protein kinase kinase, EMBO Rep. 6 (2005) 251-255.

[15] S. Yamashita, N. Mochizuki, Y. Ohba, M. Tobiume, Y. Okada, H. Sawa, K. Nagashima, M. Matsuda, CalDAG-GEFIII Activation of Ras, R-Ras, and Rap1, J. Biol. Chem. 275 (2000) 25488-25493.

[16] A. Eger, K. Aigner, S. Sonderegger, B. Dampier, S. Oehler, M. Schreiber, G. Berx, A. Cano, H. Beug, R. Foisner, DeltaEF1 is a transcriptional repressor of E-cadherin and regulates epithelial plasticity in breast cancer cells, Oncogene 24 (2005) 2375-2385.

[17] H. Miyoshi, Gene delivery to hematopoietic stem cells using lentiviral vectors, Methods Mol. Biol. 246 (2004) 429-438.

[18] B. Franke, J. W. Akkerman, J. L. Bos, Rapid Ca2+-mediated activation of Rap1 in human platelets, EMBO J. 16 (1997) 252-259.

[19] T. Yamada, M. Tsuda, Y. Ohba, H. Kawaguchi, Y. Totsuka, M. Shindoh, PTHrP promotes malignancy of human oral cancer cell downstream of the EGFR signaling, Biochem. Biophys. Res. Commun. 368 (2008) 575-581. 
[20] A. Honda, S. R. Adams, C. L. Sawyer, V. Lev-Ram, R. Y. Tsien, W. R. Dostmann, Spatiotemporal dynamics of guanosine 3',5'-cyclic monophosphate revealed by a genetically encoded, fluorescent indicator, Proc. Natl. Acad. Sci. U.S.A. 98 (2001) 24372442.

[21] Y. Ohba, N. Mochizuki, S. Yamashita, A. M. Chan, J. W. Schrader, S. Hattori, K. Nagashima, M. Matsuda, Regulatory proteins of R-Ras, TC21/R-Ras2, and M-Ras/R-Ras3, J. Biol. Chem. 275 (2000) 20020-20026.

[22] G. E. Davis, D. R. Senger, Endothelial extracellular matrix: biosynthesis, remodeling, and functions during vascular morphogenesis and neovessel stabilization, Circ. Res. 97 (2005) 1093-1107.

[23] M. Potente, C. Urbich, K. Sasaki, W. K. Hofmann, C. Heeschen, A. Aicher, R. Kollipara, R. A. DePinho, A. M. Zeiher, S. Dimmeler, Involvement of Foxo transcription factors in angiogenesis and postnatal neovascularization, J. Clin. Invest. 115 (2005) 2382-2392.

[24] R. Sekido, K. Murai, J. Funahashi, Y. Kamachi, A. Fujisawa-Sehara, Y. Nabeshima, H. Kondoh, The delta-crystallin enhancer-binding protein delta EF1 is a repressor of E2-boxmediated gene activation, Mol. Cell. Biol. 14 (1994) 5692-5700.

[25] D. M. Roberts, A. L. Anderson, M. Hidaka, R. L. Swetenburg, C. Patterson, W. L. Stanford, V. L. Bautch, A vascular gene trap screen defines RasGRP3 as an angiogenesis-regulated gene required for the endothelial response to phorbol esters, Mol. Cell. Biol. 24 (2004) $10515-10528$.

[26] R. H. Adams, K. Alitalo, Molecular regulation of angiogenesis and lymphangiogenesis, Nat. Rev. Mol. Cell Biol. 8 (2007) 464-478. 


\section{FIGURE LEGENDS}

Fig. 1. TCF8 activates R-Ras to negatively regulate angiogenesis. (A) HUVECs were transfected with si Control or si TCF8. After $48 \mathrm{~h}$, the cells were trypsinized, plated on type I collagencoated dishes for $1 \mathrm{~h}$, lysed, and incubated with GST-Ral-GDS to determine the level of GTPbound R-Ras (upper panel). The second, third, and forth panels show the expression levels of total R-Ras, TCF8, and $\beta$-actin, respectively. (B) 293T cells were transfected with an expression vector for Flag-TCF8 or a control vector along with that for R-Ras. Active R-Ras was measured as described above. (C) HUVECs were infected with lentivirus vectors for R-Ras V38 or mock viruses, further transfected with si Control or si TCF8. The levels of protein expression are shown. (D and E) The cells were then subjected to tube formation assay on Matrigel. The number of tubes was quantitated. * $P=0.00353$; ** $P=0.00898 ; * * *, P=0.00847 ; \dagger, P=$ 0.230 (D). Representative images are also shown (E).

Fig. 2. (A and B) TCF8 activates R-Ras via C-terminal ZF-domains. (A) Schematic representation of TCF8 deletion mutants. FL, full length; NZF and CZF, amino- and carboxylterminal ZF domains; HD, homeo-like domain. (B) Activities of R-Ras in 293T cells expressing HA-tagged TCF8 and its deletion mutants were determined by pull-down assay. (C and D) Transcriptional regulation of R-Ras regulators is dispensable for the TCF8-mediated R-Ras activation. (C) HUVECs were prepared as in Fig. 1A. The expression levels of GEFs and GAPs indicated at the bottom were determined by RT-PCR. (D) 293T cells prepared as in Fig. 1B were processed as in $(\mathrm{C})$.

Fig. 3. TCF8 binds to CalDAG-GEFIII in the cytosol. (A) 293T cells were transfected with expression vectors as indicated on the top, lysed in lysis buffer, and immunoblotted by antibodies described at the bottom. (B) HUVEC lysates were immunoprecipitated (IP) with normal rabbit serum (NRS) or anti-CalDAGIII antiserum. The immunoprecipitates were immunoblotted (IB) with anti-TCF8 followed by re-probing with anti-CalDAG-GEFIII. (C) 293T cells were transfected as indicated at the top. After $24 \mathrm{~h}$, the levels of GTP-R-Ras were determined. (D) 
Localization of TCF8 and its deletion mutants in $293 \mathrm{~T}$ cells were determined by immunofluorescence. (E) HeLa cells expressing TCF8-CFP and YFP-CalDAGIII were subjected to microscopic analysis, and an image representing corrected FRET (FRET ${ }^{c}$ ) is shown. 
Figure 1

A

B

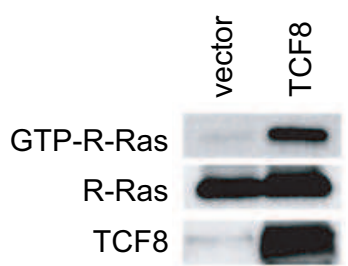

C

D
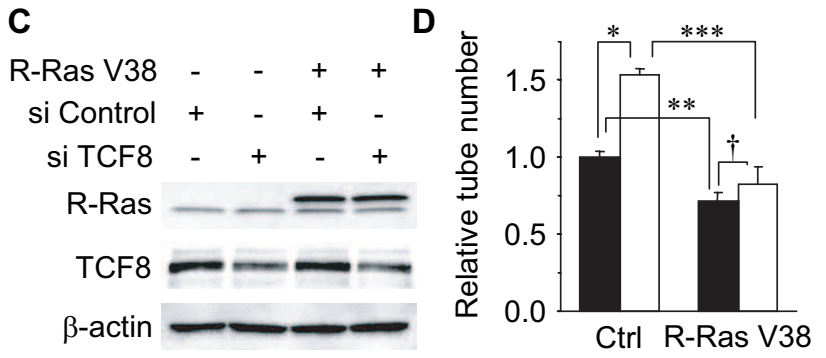

E

$\square$ si Control $\square$ si TCF8

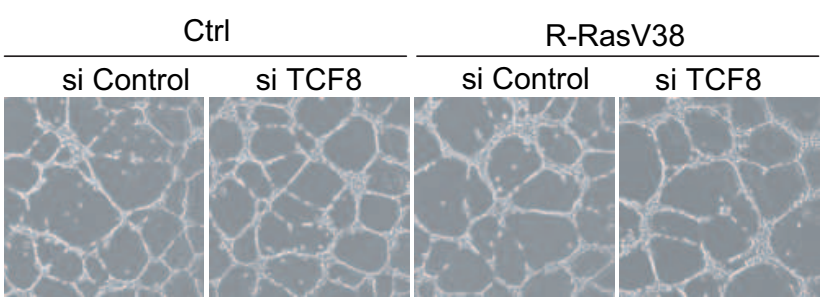

Figure 1. Inuzuka, T., et al. 
Figure 2

A

FL: \begin{tabular}{l|l|l|l|l|l|}
1 & 1124 \\
\hline
\end{tabular}

NZF: $\square$

$\mathrm{HD}$ :

509

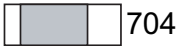

HDCZF:

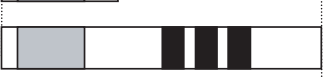

CZF: $685 \square$

Zinc-finger $\square$ Homeo-like

B

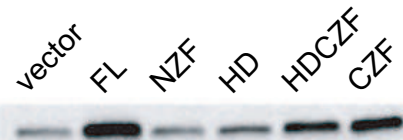

GTP-R-Ras

$\mathrm{R}$-Ras

TCF8

mutants

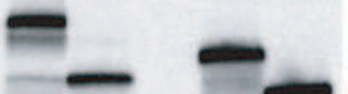

C

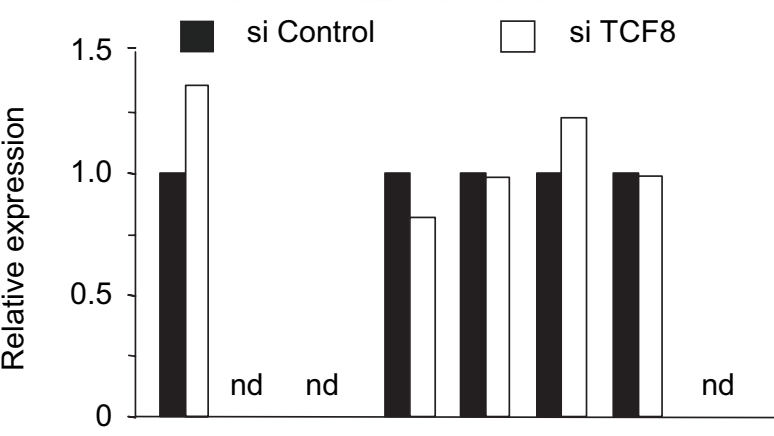

D

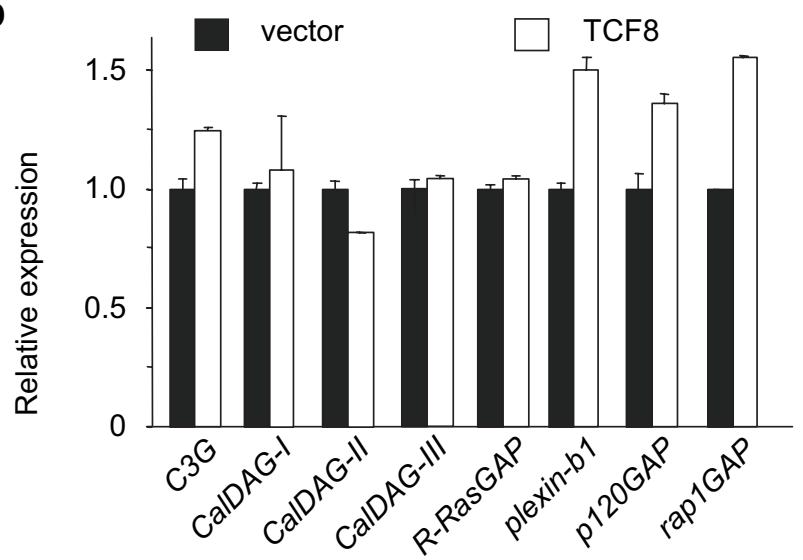

Figure 2. Inuzuka, T., et al. 
Figure 3

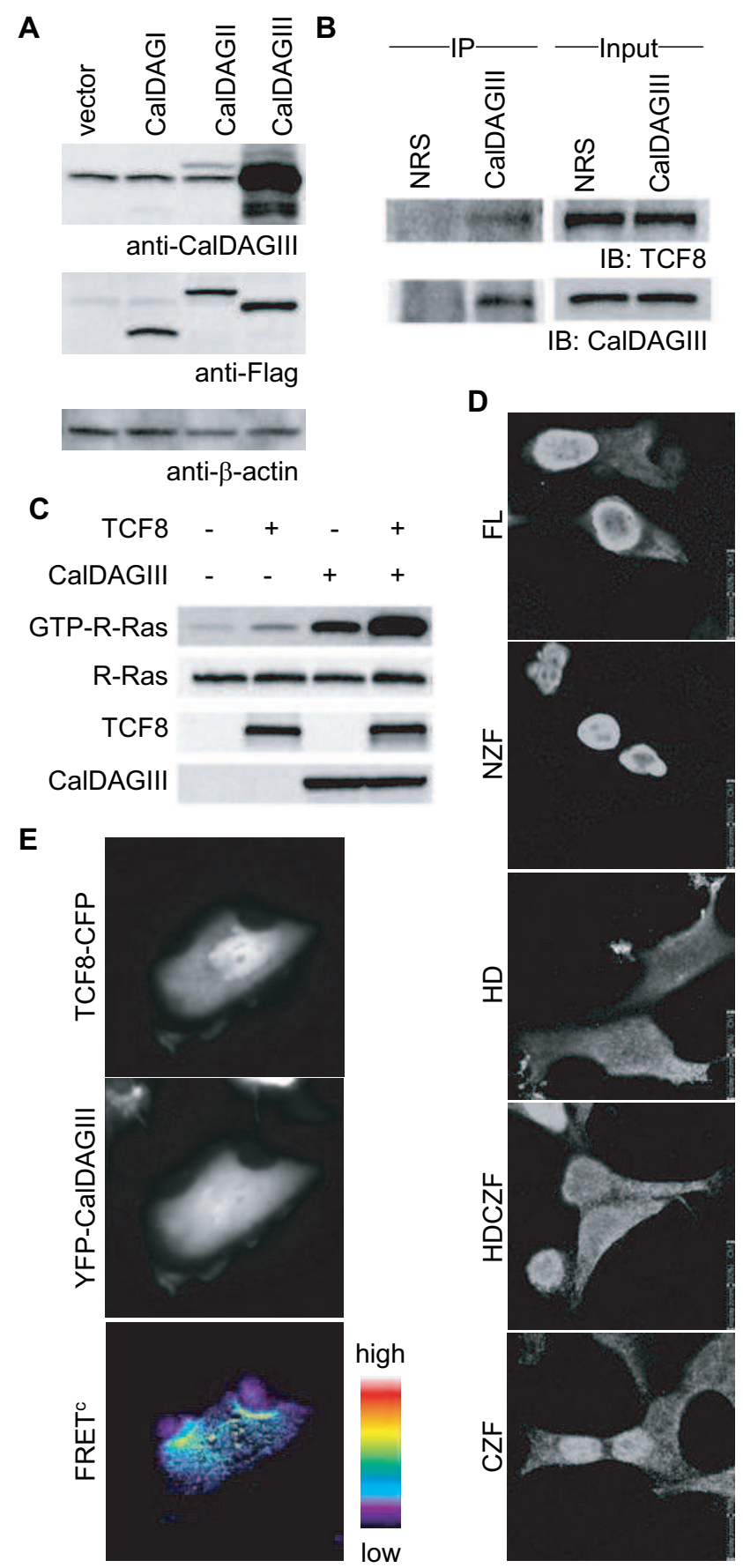

Figure 3. Inuzuka, T., et al. 
Supplementary Table 1. Sequence for primers used in the following experiments

\begin{tabular}{l|ll}
\hline \multicolumn{2}{c}{ 'semi quantitative RT-PCR } \\
\hline \multicolumn{1}{c}{ Target } & \multicolumn{2}{c}{ Forward } \\
\hline GAPDH & 5'-GAAATCCCATCACCATCTTCCAGG-3' & 5'-CATGTGGGCCATGAGGTCCACCAC-3' \\
R-RasGAP & 5'-CCCCAGTTCGATGAAGTGTT-3' & 5'-CTCCCAGGAATTCATCTCCA-3' \\
plexin b1 & 5'-TCTGCTCAGTGACCTGGTTG-3' & 5'-GTGTATTTGGCCTTGCCTGT-3' \\
p120 GAP & 5'-AGCGAAAAACGAGCTACCAA-3' & 5'-CGAAGGCGTTTATTGGATGT-3' \\
rap1GAP & 5'-CTACCGGAAGCACTTTCTCG-3' & 5'-CACACACCAACTTTGCCATC-3' \\
CalDAGI & 5'-TCTACCAACAATCCCGGAAG-3' & 5'-GGGACGCTGTCTATGCTGAT-3' \\
CalDAGII & 5'-TCTGGAGTGTCTCCCAAACC-3' & 5'-AGGCCCAGCTTGGAATAGAT-3' \\
CalDAGIII & 5'-GCTCTCCGTTACCCTGAGTG-3' & 5'-TTCTAGGCTCCAGCACCAGT-3' \\
C3G & 5'-GGAAGGCGTGAACTCAGAAG-3' & 5'-GATCTCTACCTCGCGGTCTG-3' \\
\hline
\end{tabular}

real time RT-PCR - SYBRGreen-

\begin{tabular}{l|ll}
\hline \multicolumn{1}{c|}{ Target } & \multicolumn{1}{c}{ Forward } & Reverse \\
\hline R-RasGAP & 5'-CCCCAGTTCGATGAAGTGTT-3' & 5'-CTCCCAGGAATTCATCTCCA-3' \\
plexin b1 & 5'-TCTGCTCAGTGACCTGGTTG-3' & 5'-GTGTATTTGGCCTTGCCTGT-3' \\
p120 GAP & 5'-AGCGAAAAACGAGCTACCAA-3' & 5'-CGAAGGCGTTTATTGGATGT-3' \\
rap1GAP & 5'-CTACCGGAAGCACTTTCTCG-3' & 5'-CACACACCAACTTTGCCATC-3' \\
CalDAGI & 5'-TCTACCAACAATCCCGGAAG-3' & 5'-GGGACGCTGTCTATGCTGAT-3' \\
CalDAGII & 5'-TCTGGAGTGTCTCCCAAACC-3' & 5'-AGGCCCAGCTTGGAATAGAT-3' \\
CalDAGIII & 5'-TCCATGACAAAGCAGCAAAG-3' & 5'-CCATCCTCACCATCCTGTCT-3' \\
C3G & 5'-GGAAGGCGTGAACTCAGAAG-3' & 5'-GATCTCTACCTCGCGGTCTG-3' \\
\hline
\end{tabular}


